\title{
Réussir le développement des communautés rurales : dix conditions gagnantes
}

\author{
Bruno Jean ${ }^{1}$ \\ Université du Québec à Rimouski
}

Les territoires ruraux sont particulièrement sensibles aux transformations économiques et sociales actuelles comme la mondialisation ou la nouvelle économie du savoir. Les restructurations et les adaptations y sont probablement plus importantes que dans le reste de la société, ce qui questionne la cohésion sociale des petites collectivités constituant la trame des milieux ruraux. Par ailleurs, les ruraux ne sont pas seulement des producteurs. Les ruraux occupent et aménagent le territoire; par là, ils exercent une fonction géopolitique essentielle car ils affirment la souveraineté politique sur ce même territoire. Cette occupation du territoire par les populations rurales étant menacée par la dynamique économique actuelle, le politique est justifié d'intervenir.

Un tel contexte aide à comprendre pourquoi la ruralité est devenue une réalité davantage reconnue par les pouvoirs publics du Québec avec la Politique nationale de la ruralité annoncée le 6 décembre 2001. Celle-ci fournit un cadre d'action largement basé sur la capacité des populations rurales à se mobiliser pour utiliser avec intelligence et efficience les sommes disponibles à l'échelle d'un niveau privilégié d'intervention, la municipalité régionale de comté (MRC). Mais cette politique laisse entières un certain nombre de questions sur la compréhension des dynamiques sociales et économiques qui soutiennent le développement rural d'aujourd'hui et sur les moyens de faire en sorte que plusieurs communautés rurales en dévitalisation s'inscrivent dans une perspective de développement durable.

Le développement durable ou soutenable, et je dirais personnellement approprié, des communautés rurales suppose de réunir plusieurs conditions dont chacune est nécessaire mais non suffisante. On peut parler des conditions gagnantes pour contrer les processus de dévitalisation rurale ${ }^{2}$. Un jour, j'ai réalisé que la dizaine de conditions nécessaires que certaines de mes recherches, ou celles de mes collègues, avaient permis de mettre à jour pouvaient être déclinées dans un mode prescriptif comme les dix commandements de Dieu selon la religion chrétienne. Ces dix conditions gagnantes peuvent être énoncées de la manière suivante: reconnaître qu'on peut apprendre de la ruralité; protéger la sociodiversité à laquelle contribue la ruralité; favoriser une approche de développement rural durable; reconnaître la multifonctionnalité de la nouvelle économie rurale; mobiliser le capital social et les infrastructures sociales entrepreneuriales; soutenir le développement des capacités des communautés rurales; soutenir l'émergence d'une nouvelle gouvernance rurale citoyenne; rendre intelligible l'interdépendance rurale-urbaine; soutenir une nouvelle solidarité rurale-urbaine par une taxe pédagogique et mettre en œuvre une politique publique appropriée.

\section{Reconnaître qu'on peut apprendre de la ruralité}

Dans l'opinion publique comme dans les milieux scientifiques, on s'accorde pour penser que le monde rural se caractérise par un fort attachement aux valeurs traditionnelles et qu'il manque de sens de l'initiative, de créativité et d'innovation. Le retard de la ruralité sur la route du développement se trouve par le fait même expliqué. Il faut changer notre point de vue sur la ruralité, lui-même empreint d'une sorte d'ethnocentrisme urbain. Les milieux ruraux sont plutôt des milieux innovateurs, l'innovation y prenant parfois des allures de nécessité vitale, et s'ils sont tels, leurs expériences peuvent être utiles et toute la société peut en tirer des enseignements. Une première condition gagnante pour assurer le développement rural émerge alors ici, soit reconnaître qu'on peut apprendre de la ruralité. C'est en réalisant l'Université rurale québécoise qu'on a compris l'importance d'une telle condition gagnante qui repose aussi sur l'idée de plus en plus reconnue que le déve- 
loppement doit s'appuyer sur les savoirs d'expérience et les savoir-faire locaux. Et si nous pouvons apprendre de la ruralité, c'est que les ruraux ont déjà eux-mêmes appris dans trois grands domaines qui, s'ils sont harmonisés, peuvent conduire à un développement durable: 1) la mise en valeur de leur environnement naturel; 2) la gestion de leur développement économique; 3) l'aménagement de leur organisation sociale.

\section{C'est en réalisant l'Université rurale québécoise qu'on a compris l'importance d'une telle condition gagnante qui repose aussi sur l'idée de plus en plus reconnue que le développement doit s'appuyer sur les savoirs d'expérience et les savoir-faire locaux. Et si nous pouvons apprendre de la ruralité, c'est que les ruraux ont déjà eux-mêmes appris dans trois grands domaines qui, s'ils sont harmonisés, peuvent conduire à un développement durable: 1) la mise en valeur de leur environnement naturel; 2) la gestion de leur développement économique; 3) I'aménagement de leur organisation sociale.}

Cette créativité des ruraux s'est traduite par un ensemble d'innovations sociales et institutionnelles qu'ils se sont avérés capables de mettre en place. Ces innovations sociales sont nombreuses mais mal connues et c'est certainement un des mandats majeurs de l'Université rurale québécoise d'en rendre compte pour le bénéfice de toute la société. Cela non seulement leur permet de s'adapter aux changements, mais aussi d'offrir à toute la société des modes de vie alternatifs, différents, d'autres valeurs ainsi que des modèles organisationnels et institutionnels inédits qui se répandent souvent dans le reste de la société. On oublie trop souvent, par exemple, que les «caisses populaires» sont nées en milieu rural et que des concepts comme l'autogestion y ont été expérimentés d'une manière originale qui a enrichi cette notion elle-même. Par ailleurs, comme on le voit souvent, les ruraux sont aussi capables d'adapter des institutions sociales et économiques qui sont pensées pour des sociétés urbaines et imposées à l'ensemble de la société.

Les conséquences d'une telle prise de position sont nombreuses et importantes. Au lieu de voir les ruraux comme partie du problème, il faut plutôt les voir comme faisant partie de la solution. Le développement rural n'est pas un objectif qui peut être atteint par des interventions et une expertise externes mais une finalité qui est la responsabilité propre des ruraux. En d'autres termes, il faut aussi comprendre que les ruraux sont tout à fait capables, comme d'autres, de s'inscrire dans des processus d'apprentissage social qui vont soutenir leur propre démarche de développement.

\section{Protéger la socio-diversité à laquelle contribue la ruralité}

Avec la montée de la pensée écologique, on a pris conscience de la nécessité d'actions volontaristes pour protéger les espèces menacées d'extinction et préserver ainsi la richesse des écosystèmes. Le maintien de la biodiversité est devenu une valeur universellement partagée. Les petits villages ruraux, à l'ombre des métropoles qui leur font d'ailleurs un peu trop d'ombrage ces derniers temps, font partie du patrimoine social, culturel, architectural et paysager. Plusieurs conventions internationales sur la protection du patrimoine ont été négociées par nos gouvernements qui ne semblent pas conscients des accords qu'ils signent et de leurs conséquences locales. La mise en valeur de ce riche patrimoine sur lequel prospère une industrie récréotouristique en plein développement devrait donc s'inscrire dans la reconnaissance de la nécessité de préserver la socio-diversité dans le monde humain comme le concept de biodiversité nous invite à le faire pour le monde animal et végétal.

Une certaine pensée simplificatrice voudrait nous faire croire que le modèle urbain convient bien aux campagnes. Les petites collectivités rurales ont démontré à maintes reprises leur vitalité sociale, attestée par de nombreuses mesures de l'occurrence plus faible de diverses pathologies sociales. Les petites collectivités ont aussi démontré leur viabilité économique même avec des taux plus élevés de chômage liés à la saisonnalité des activités de mise en valeur des ressources naturelles. Finalement, elles sont un lieu d'exercice de la citoyenneté, comme on dit aujourd'hui, ou le lieu d'expression d'une société civile capable de gérer son existence collective avec le maintien d'un tissu de liens sociaux assurant une bonne cohésion sociale.

Que des petites collectivités, si elles le désirent, se regroupent avec une ville proche, cela peut s'avérer mutuellement bénéfique pour les deux. Quand une certaine pensée unique, encore une fois urbanisante, 
parle des bienfaits de ces fusions, qu'est-ce qu'on attend pour aller voir les effets de la fusion de plusieurs petits villages autour de la ville de Gaspé il y a plus de trente ans? On le sait tous, et comme dirait une certaine opinion publique trop contente de s'inscrire dans le vieux modèle charitable, la Gaspésie fait encore pitié.

\section{Favoriser une approche de développement rural durable}

La réussite du développement des communautés rurales nécessite une approche globale et intégrée où devront se concerter logiquement les recherches visant à la fois l'identification des opportunités économiques, des contraintes écologiques et des attentes sociales. Car le développement rural durable suppose de réussir l'harmonisation entre ces trois dimensions: le respect de l'environnement, la recherche d'une viabilité économique et la réponse aux attentes des populations.

Mais nous sommes actuellement devant un paradoxe. La ruralité et les ruraux, plus proches de l'environnement, du moins l'environnement naturel, seraient devenus avec les modes de gestion et de production actuels, incapables de bien s'occuper de l'environnement. Pourtant, il devient impératif de penser à des «systèmes ruraux durables» basés sur la reconnaissance des réalités environnementales, comme c'est déjà le cas avec les conseils de bassin (Rivière Rimouski par exemple) et les clubs agroenvironnementaux qui encadrent maintenant des milliers de producteurs agricoles. Ces systèmes seront aussi basés sur l'autoresponsabilisation des producteurs, sans sacrifier le principe de précaution mais sans une judiciarisation infinie des pratiques agricoles.

Des films comme L'erreur boréale du chanteur Richard Desjardins et Bacon le film, commandité par l'Union paysanne, sont venus jeter un doute sérieux sur la prétendue capacité des ruraux à gérer leur environnement. Mais ces cris d'alarme visant des objectifs de sensibilisation d'un public urbain passent sous silence un nombre considérable d'efforts des ruraux pour une gestion raisonnée des terres comme des forêts qui commencent à donner des résultats tangibles. Pour nous, il est clair que l'approche du développement durable est une voie dans laquelle il faut s'engager résolument. Les ruraux le pensent aussi. Si on prend la peine de les écouter, on verra combien ils sont inquiets des pratiques des grandes sociétés intervenant en milieu rural sur lesquelles ils n'ont guère de pouvoir. Mais on assiste à

un rapprochement du regard urbain sur les réalités rurales. La question environnementale est une question qui intéresse toute la société. Comme il existe des problèmes environnementaux en milieu rural, les populations urbaines s'y intéressent et cela ne laissera pas indifférents les acteurs politiques. Si la question environnementale devient une nouvelle chance pour le monde rural de recevoir de l'attention de toute la société, il est à souhaiter que cela devienne l'occasion de nouer un dialogue rural-urbain constructif car, et c'est une intime conviction pour nous, tout processus de développement de la ruralité québécoise passe par une solidarité des villes avec les campagnes.

La question environnementale est une question qui intéresse toute la société. Comme il existe des problèmes environnementaux en milieu rural, les populations urbaines s'y intéressent et cela ne laissera pas indifférents les acteurs politiques. Si la question environnementale devient une nouvelle chance pour le monde rural de recevoir de l'attention de toute la société, il est à souhaiter que cela devienne l'occasion de nouer un dialogue rural-urbain constructif car, et c'est une intime conviction pour nous, tout processus de développement de la ruralité québécoise passe par une solidarité des villes avec les campagnes.

L'identification des conditions gagnantes suppose, selon le modèle de développement soutenable, d'identifier celles qui touchent à ces trois grandes dimensions et à leur harmonisation. Au plan environnemental, s'il faut bien saisir les potentialités écologiques d'un territoire, il faut aussi rappeler que ce processus n'est pas neutre, que la ressource n'existe pas en dehors du regard que l'on porte sur elle et que, finalement, ce n'est pas nécessairement la ressource qui crée le projet mais c'est le projet qui crée la ressource. Au plan économique, le développement rural implique une préoccupation constante de faisabilité économique, soit l'existence de marchés rémunérateurs pour la production rurale. Par contre, c'est souvent au plan social que nos analyses sont faibles car les opportunités écologiques et économiques, pour se concrétiser, doivent rencontrer les attentes des populations et de leurs communautés pour s'inscrire dans un développement local ou régional. 


\section{Reconnaître la multifonctionnalité de la nouvelle économie rurale}

Ces derniers temps, on parle beaucoup de la «nouvelle économie rurale» pour désigner les mutations qui concernent cette économie avec l'arrivée des NTIC, ces nouvelles technologies de l'information et de la communication. Sans contester l'importance d'un tel phénomène dont nous pouvons discuter ici, il ne faudrait pas oublier les fonctions plus classiques ou traditionnelles de la ruralité qui ne sont pas encore bien reconnues. Pour les désigner, la notion de multifonctionnalité des territoires ruraux ou de l'agriculture est en train de devenir un concept fort populaire qui fait apparaître dans des habits neufs une réalité ancienne du monde rural, soit les différentes fonctions qu'il exerce.

Par exemple, un agriculteur produit de la viande ou du lait. Mais en même temps, il aménage une parcelle de territoire qui contribue à créer un paysage rural qui bénéficie à l'industrie touristique tout en contribuant au maintien d'un écosystème et de ses ressources naturelles. Dans ce cas, trois fonctions sont exécutées en même temps, la fonction économique de production et création d'emploi, la fonction environnementale de protection et valorisation d'un écosystème, et une fonction sociale ou culturelle, la création d'un paysage ayant de la valeur, en plus probablement du maintien de certaines traditions agricoles faisant aussi partie de la culture rurale locale. Si, en plus de faire du lait, la même ferme recevait des touristes dans le cadre d'une activité d'hébergement à la ferme, les économistes parleraient alors de joint product au sens où deux produits ayant une valeur sont générés par la même entreprise, la production laitière et l'accueil touristique et où chacun de ces produits ne peut être réalisé isolément.

\section{Les multiples fonctions (multifonctionnalité) des territoires ruraux}

- La fonction de production : fournir aux consommateurs des produits sains et de qualité, des produits dont l'origine et les conditions de production sont connues.

- La fonction territoriale : occuper les territoires, gérer l'espace, préserver les paysages et les ressources naturelles.

- La fonction sociale : contribuer à l'emploi, à l'animation du milieu rural, produire des services collectifs.
La reconnaissance actuelle de la multifonctionnalité repose sur les tentatives par les organisations rurales de faire admettre que ce type d'économie produit, dans un même mouvement, des biens marchands et d'autres sortes de biens non marchands qui sont tout aussi nécessaires pour le bien-être de nos sociétés, soit la protection des écosystèmes naturels et la création de paysages ruraux humanisés ayant de la valeur aux yeux de la population urbaine. Comme ce sont des biens non marchands qu'il importe de produire et qui ne sont pas reconnus, validés et rémunérés dans le cadre de la régulation marchande, il faut bien envisager le versement de subventions, de subsides aux agriculteurs et aux ruraux à même l'économie publique pour qu'ils puissent continuer à assurer ces fonctions, au-delà de la fonction stricte de production.

Mais dans le cadre des discussions autour de la libéralisation du commerce agricole international à l'OMC (Organisation mondiale du commerce), les Américains ne veulent pas reconnaître la multifonctionnalité des régions rurales, laquelle, selon les Européens et les Japonais, autorise les gouvernements à intervenir dans le secteur agricole en y maintenant des politiques de soutien au nom de la protection de l'environnement et du développement local et régional des territoires en difficulté. Le Canada suit le raisonnement américain car les producteurs de céréales de l'Ouest se pensent victimes des entraves au commerce et aisément capables de conquérir les marchés mondiaux. L'agriculture du Québec ne peut avoir une telle prétention et sert bien son marché domestique. Mais la position politique du Québec devrait être la défense de la multifonctionnalité, ce qui lui permettra de maintenir divers programmes d'intervention en faveur des régions en difficulté et de leurs nombreuses petites collectivités rurales dites en restructuration. Sans la reconnaissance de la multifonctionnalité à l'ordre du jour de la prochaine ronde de négociations à l'OMC, ces communautés rurales risquent de perdre une autre condition gagnante pour réussir leur développement, soit les politiques de soutien du secteur public dont elles ont et auront toujours besoin pour lutter efficacement contre les tendances naturelles de l'économie qui les condamnent à un déclin constant.

\section{Mobiliser le capital social et les infrastructures sociales entrepreneuriales}

Les chercheurs en développement rural et les décideurs publics sont confrontés au constat suivant: des com- 
munautés rurales gagnent et d'autres perdent... semblant être enfermées dans le cercle vicieux de la dévitalisation. Des facteurs traditionnels de développement comme la dotation en ressources et la localisation s'avèrent insatisfaisants pour expliquer le développement dans plusieurs localités. D'où la nécessité de comprendre le rôle des facteurs intangibles de développement comme certaines «capacités» à se développer propres aux communautés, tel le capital social, une ressource et une production sociale intangible qui pourrait s'avérer un facteur de développement de communautés rurales soutenables.

Le concept de «capital social» réfère aux différentes «capacités » (collectives) relatives à la vitalité sociale et la viabilité économique de toute communauté. Ses trois principales dimensions (potentiellement mesurables) sont:

- la diversité symbolique ou le degré d'ouverture de la population aux personnes différentes, aux idées ou aux comportements;

- la capacité d'investissement-réinvestissement, soit de mobiliser des ressources (temps, argent, bénévolat, etc.) pour des activités de la communauté et la capacité de travailler ensemble;

- la capacité d'établir des réseaux et des liens avec l'extérieur pour notamment en obtenir des avantages des gouvernements.

La richesse du capital social varie en fonction du type de communauté: en croissance ou en déclin. Un capital social riche et diversifié, dont plusieurs théories du développement local postulent qu'il est un facteur nécessaire de développement, semble être davantage présent dans les localités rurales en croissance (leading). Dans les municipalités en dévitalisation (lagging), son niveau serait significativement plus bas.

L'hypothèse des effets structurants et déterminants du capital social dans le développement des communautés rurales soutenables est attestée par les travaux de l'Initiative de recherche sur la Nouvelle Économie Rurale $^{3}$ de la Fondation canadienne pour la revitalisation rurale. Ces travaux de recherche apportent une contribution à la validation des théories du développement local qui mettent l'accent sur des facteurs intangibles ou immatériels de développement comme le «capital social» ou l'apprentissage social, l'innovation, la gouvernance, etc. plutôt que sur les facteurs structurels (dotation en ressources, localisation, investissement de l'extérieur) des théories «néo-classiques » du développement.

\section{Soutenir le développement des capacités des communautés rurales}

La différence entre une collectivité en déclin et une collectivité prospère ne tiendrait donc pas seulement aux facteurs traditionnels (dotation en ressources naturelles, capitaux, localisation, etc.) mais aussi à un facteur immatériel: les «capacités» de se développer de la communauté elle-même. Le «renforcement des capacités ${ }^{4}$ peut être alors défini comme un processus par lequel les individus, les groupes, les organisations et les sociétés renforcent leurs habiletés à identifier et à relever les défis de développement sur une base durable.

Comment reconnaître une communauté rurale aux capacités développées? C'est une communauté active qui prend des initiatives pour résoudre ses problèmes ellemême; c'est une communauté qui prospère en se basant sur les compétences et les ressources locales et qui est capable de prendre en compte les attentes de ses citoyens. C'est aussi une communauté forte alimentée par un esprit d'entreprise mais aussi par le volontariat et l'entraide et finalement, c'est une communauté avec des structures appropriées de gouvernance (synergie entre les secteurs public, privé et communautaire). Le renforcement des capacités est lié aux nouvelles approches de développement local s'appuyant sur le concept de «capital social» qui sera présenté dans le point suivant.

Le développement local, probablement comme d'autres pratiques sociales, est susceptible de s'apprendre en se faisant et en s'inspirant de l'expérience des autres. Ce qui est fondamentalement nouveau ici, c'est la nécessité de faire reconnaître cette nouvelle mission des communautés locales et de leurs instances politiques et qui, tout compte fait, n'est rien d'autre que la fonction «développement». Les collectivités rurales doivent maintenant s'occuper de leur développement et ne plus l'attendre par l'action des intervenants publics ou privés. Malgré diverses expérimentations intéressantes, une véritable pédagogie du développement local reste encore à inventer. Car il est clair que la maîtrise du développement passe par des actions de formation et par l'élévation du niveau général d'éducation des popu- 
lations locales comme par exemple l'apprentissage par les leaders locaux de divers savoirs et techniques reliés à l'animation et la gestion de projet.

Le développement local, probablement comme d'autres pratiques sociales, est susceptible de s'apprendre en se faisant et en s'inspirant de l'expérience des autres. Ce qui est fondamentalement nouveau ici, c'est la nécessité de faire reconnaître cette nouvelle mission des communautés locales et de leurs instances politiques et qui, tout compte fait, $n$ 'est rien d'autre que la fonction " développement ". Les collectivités rurales doivent maintenant s'occuper de leur développement et ne plus l'attendre par I'action des intervenants publics ou privés.

Un nouveau champ de connaissance et d'action s'ouvre ici et les savoirs validés, comme l'évaluation des diverses expériences, ne sont pas légions. Pourtant, plusieurs initiatives commencent à dégager des enseignements qui permettent de comprendre les processus qui conduisent au renforcement des capacités de développement des communautés. Un modèle privilégié est celui qui repose sur l'embauche d'un agent rural accompagnant la collectivité et ses acteurs dans une démarche de développement local. Après avoir suivi une expérience grandeur nature dans deux municipalités dans le cadre d'un projet-pilote intitulé «Opération villages en mouvement», bien que nos conclusions ne soient pas encore rendues publiques, nous nous posons des questions sur la pertinence du rattachement administratif (c'est-à-dire avec un lien d'emploi) de l'agent rural avec la municipalité locale. On pourrait penser que la même personne pourrait être au service d'une, deux ou trois municipalités rurales dans le cadre des opérations du CLD ce qui la rendrait moins vulnérable aux changements rapides d'agenda des instances politiques locales.

\section{Soutenir l'émergence d'une nouvelle gouvernance rurale citoyenne}

La notion de gouvernance est passée dans le langage scientifique et journalistique ces dernières années, et même dans le discours de certaines organisations comme Solidarité rurale du Québec. La notion de gouvernance a été popularisée par les agences des Nations-Unies, comme le PNUD, le Programme des Nations-Unies pour le développement, sous le concept de «bonne gouvernance» pour signifier la mise en place dans les pays en développement de meilleures pratiques des administrations publiques, soit une saine gestion des finances publiques d'une part, et une plus grande démocratisation des régimes politiques comme tels d'autre part.

Mais le concept de gouvernance, sans son qualificatif, permet de décrire une réalité sensiblement différente, soit le mode spécifique d'organisation d'un gouvernement local, régional ou national, mode qui se révèle dans la manière dont un gouvernement aménage ses rapports avec les autres forces socio-économiques agissantes dans la société 5 . La notion de gouvernance s'inscrit dans ce que les scientifiques appellent un nouveau paradigme dans lequel elle appartient à un univers conceptuel plus large, avec d'autres concepts inter-reliés comme celui de société civile, de secteur communautaire, de concertation ou de partenariat.

\section{La nouvelle gouvernance rurale désigne alors cette nouvelle manière d'exercer le pouvoir et de prendre des décisions et elle met en scène trois grandes catégories d'acteurs: le pouvoir municipal, le pouvoir des acteurs économiques privés ayant une présence locale et le pouvoir de la société civile ou des organismes communautaires qui en sont I'incarnation en quelque sorte.}

Pour nous, s'agissant des réalités rurales, la gouvernance désigne les arrangements institutionnels inédits qui font que les gouvernements locaux exercent un pouvoir efficient, c'est-à-dire un pouvoir qui prend des décisions qui ont une prise sur le destin des communautés locales. On parle de nouvelle gouvernance rurale, mais on pourrait en dire autant de la gouvernance urbaine, pour désigner le fait qu'on soit en train de vivre la mise en place de cette nouvelle gouvernance rurale en certains endroits où les gouvernements locaux sont sortis de leur champ de juridiction traditionnel imposé par les gouvernements supérieurs (les gouvernements locaux étant des «créatures» des provinces, comme on le voit bien avec les débats sur les fusions) pour se donner des moyens d'intervenir dans ce qu'on pourrait appeler le «développement». La nouvelle gouvernance rurale désigne alors cette nouvelle manière d'exercer le pouvoir et de prendre des décisions et elle met en scène 
trois grandes catégories d'acteurs : le pouvoir municipal, le pouvoir des acteurs économiques privés ayant une présence locale et le pouvoir de la société civile ou des organismes communautaires qui en sont l'incarnation en quelque sorte.

Dans les modes de gouvernance traditionnelle, ces trois grandes forces présentes dans les communautés locales poursuivaient chacune leur finalité sans trop d'interaction dans un monde économique et social perçu comme relativement stable. Mais dans le monde d'aujourd'hui, les communautés rurales relativement homogènes caractérisant le Québec d'autrefois vont faire place à un monde compétitif où il y aura des communautés rurales (et des régions) qui gagnent et d'autres qui perdent. Pour être du côté des communautés gagnantes, il est apparu, de manière intuitive (la théorie est arrivée bien après), qu'il fallait faire de la concertation et du partenariat entre le secteur public, le secteur privé et le secteur communautaire. À rebours, on a constaté que les «villages prospères ${ }^{6}$ étaient justement ceux où une analyse approfondie de la dynamique locale montrait que ce partenariat entre les différentes forces locales s'était mis en place pour produire des arrangements institutionnels locaux inédits... et qui vont bien au-delà des traditionnels incitatifs fiscaux de la municipalité pour attirer une entreprise sur son territoire.

La nouvelle gouvernance locale qui semble se mettre en place en certains endroits est cette nouvelle manière dont des communautés locales organisent la prise de décision concernant les grands domaines de la vie socioéconomique de la communauté. Par ailleurs, une telle approche implique plusieurs choses, comme le «renforcement des capacités» des communautés à gérer leurs propres affaires... et cela ouvre un vaste chantier de travail pour diverses organisations et pour des générations d'agents de développement. Par ailleurs, tout cela arrive dans un contexte où les traditionnelles théories du développement économique local sont en profond changement. Alors que les théories traditionnelles mettaient l'emphase sur des facteurs comme la localisation favorable, la présence de ressources naturelles et des investissements de l'extérieur, les nouvelles théories de développement local mettent l'accent sur des facteurs immatériels ou intangibles comme l'entrepreneurship, le partenariat, les capacités innovatrices du milieu, la présence d'une infrastructure entrepreneuriale sociale (autrement dit le capital social).
En clair, ces théories économiques reconnaissent le rôle non négligeable des facteurs sociaux dans la localisation des entreprises et leur prospérité au-delà des paramètres économiques et financiers habituels. Cela veut dire qu'une entreprise qui va créer des emplois, payer des taxes, stimuler la construction domiciliaire, etc. prendra en considération des facteurs sociaux liés à la qualité de vie dans la communauté. Car elle sait que si la vie sociale est agréable, c'est parce que le secteur communautaire anime le milieu; les travailleurs y viendront plus aisément et seront plus fidèles à l'entreprise. Dans cette nouvelle gouvernance rurale, le pouvoir municipal se sentira autorisé à engager un dialogue avec le secteur privé et à faire des investissements publics qui rendront la communauté attrayante pour une entreprise en quête d'une localisation. On peut donc faire l'hypothèse que les communautés en dévitalisation sont aussi celles où la gouvernance n'est pas appropriée, ne produisant pas les synergies et les innovations sociales et institutionnelles aptes à assurer le développement de la communauté.

En clair, ces théories économiques reconnaissent le rôle non négligeable des facteurs sociaux dans la localisation des entreprises et leur prospérité au-delà des paramètres économiques et financiers habituels. Cela veut dire qu'une entreprise qui va créer des emplois, payer des taxes, stimuler la construction domiciliaire, etc. prendra en considération des facteurs sociaux liés à la qualité de vie dans la communauté.

Il faut souligner ici qu'un nouveau champ d'études de la gouvernance rurale est actuellement en construction. Comprendre la dynamique de gouvernance propre à une communauté n'est pas une tâche facile, car la mise au jour d'une telle dynamique suppose un long travail de type anthropologique avec les acteurs-clés de la communauté dans les trois domaines (public, privé, communautaire) de la gouvernance. Ce travail est généralement facilité par l'étude de certains conflits au sein de la communauté qui amènent ses membres à prendre position et... la communauté peut ainsi se révéler à elle-même. Cela peut être un conflit interne ou un événement extérieur qui est perçu comme une menace pour les uns et une opportunité pour les autres selon la conception que l'on se donne de l'identité locale. Actuellement, dans les travaux que poursuit la Fondation canadienne pour la revitalisation rurale, avec 
son Initiative de recherche sur la «Nouvelle économie rurale», il apparaît que la notion de gouvernance s'inscrit dans cet univers conceptuel où il faut introduire le concept de «cohésion sociale», notion qui renvoie à une grande valeur éthique, à un indicateur de développement social. Car cette notion reconnaît implicitement l'existence des stratifications sociales et des antagonismes sociaux entre des groupes aux intérêts variés, et cela peut se révéler aussi dans l'analyse d'une communauté locale donnée. La cohésion sociale devient alors un objectif avoué des politiques sociales et économiques.

\section{Rendre intelligible l'interdépendance rurale-urbaine}

Les pouvoirs publics, comme l'opinion publique, se questionnent sur l'avenir de la ruralité dans la modernité avancée. Plusieurs indicateurs socio-économiques témoignent d'un déclin rural pendant que certains chercheurs surveillent l'apparition des signes d'une renaissance rurale. Il faut renverser un certain discours urbain, alimenté par les politiciens, qui se demande si les campagnes servent encore à quelque chose dans notre société et notre économie.

Notre travail académique et scientifique devrait consister à mettre en évidence les liens d'interdépendance qui unissent les régions rurales et les régions urbaines dans ce pays. Une meilleure compréhension de ces liens cachés de la part des populations urbaines fera de ces dernières les meilleurs défenseurs des politiques de soutien à la revitalisation rurale. Cette question d'une meilleure compréhension des liens d'interdépendance entre les économies rurales et urbaines dans ce pays est fondamentale. Les ruraux fournissent des biens (ressources primaires) et des services écologiques à toute la société; le prix des premiers baissent sans cesse et les seconds ne sont même pas rémunérés. Mais ils devront l'être un jour par des mécanismes à inventer. Des ressources rurales (nourriture, énergie, aménités paysagères rurales, etc.) assurent la qualité de vie des urbains. Quand les populations urbaines paient ces ressources à vil prix (avec un panier d'épicerie à $17 \%$ de leur revenu disponible) pas étonnant que les économies rurales se portent mal. Il faut alors faire en sorte que des ressources urbaines (essentiellement financières) viennent supporter un processus de revitalisation des campagnes. Une fondation ${ }^{7}$ comme la Fondation canadienne pour la revitalisation rurale est d'avis que toute la société, dans un geste de solidarité bien comprise, doit supporter une

vigoureuse politique rurale capable de réussir une revitalisation rurale.

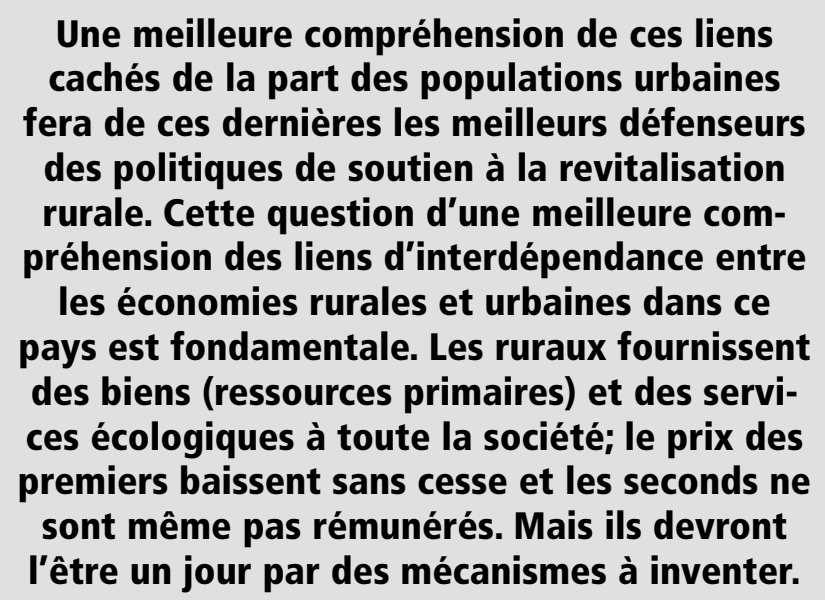

Cette condition gagnante s'impose avec une certaine urgence quand on considère la perception des ruraux à propos de leur propre situation dans la société québécoise. Lors de l'Université rurale québécoise au BasSaint-Laurent à l'automne 1999, invités à s'exprimer sur ce qu'ils feraient s'ils étaient à la tête d'un ministère du développement rural, ils ont identifié quatre enjeux majeurs qui sont à retenir ici.

1) l'accélération du phénomène de dévitalisation ou de désertification des régions rurales isolées ou éloignées des zones métropolitaines;

2) le traitement discriminatoire de la campagne par rapport à la ville;

3) la défaillance des mécanismes de soutien au processus de développement mis en place par le gouvernement;

4) la problématique du développement durable en milieu rural.

L'expérience du lointain Japon peut nous servir d'exemple ici. Ce pays se distingue par ses nombreuses zones métropolitaines. Pourtant, il s'est donné une vigoureuse politique rurale fondée sur une bonne compréhension de la symbiose entre milieux urbains et ruraux. Dans cette île surpeuplée, il est facile de concevoir que les campagnes soient des espaces à protéger, car elles assurent de nombreuses fonctions utiles à toute la société : production alimentaire certes mais bien plus, 
soit assurer la reproduction des écosystèmes naturels dont dépendent les villes pour leur alimentation en eau potable. Alors, une partie des taxes d'eau en ville retourne dans les campagnes sous forme de programmes d'aide issus d'une politique rurale bien comprise et qui rémunèrent les ruraux pour des services écologiques essentiels rendus à toute la société. Et quand les urbains partent en campagne pour leurs loisirs, ils y trouvent des campagnes vivantes; d'ailleurs, ils les trouvent tellement vivantes que les écoles des petits villages ruraux en déclin démographique sont pleines d'enfants des familles urbaines qui ont décidé de leur faire passer quelques années dans cet environnement propice à divers apprentissages. Les citoyens de Sainte-Paule, près de Matane, qui ont sauvé leur école de village de cette manière n'ont pas eu besoin d'un voyage d'études au Japon pour comprendre que les autobus scolaires circulent dans les deux sens et peuvent donc amener les petits urbains dans une école primaire rurale.

La politique rurale japonaise, comme celle en Europe, est basée sur la reconnaissance de la multifonctionnalité des territoires ruraux. Elles devraient inspirer la nôtre, celle que nous risquons d'attendre encore longtemps. Il faut faire reconnaître le rôle de la puissance publique dans le soutien aux territoires ruraux en faisant admettre que cela n'est pas une entrave au commerce agricole comme voudraient le croire les fonctionnaires de l'OMC. Une telle politique devrait aussi reposer sur un exercice pédagogique qui consisterait à mieux faire comprendre à l'ensemble de la société les liens d'interdépendance qui unissent les régions urbaines aux milieux ruraux même les plus éloignés. Un seul exemple: sans les grandes rivières qui caractérisent ces territoires, qu'en serait-il de l'industrie hydroélectrique, un fleuron de l'économie du Québec et un puissant levier de développement de toute cette société?

\section{Soutenir une nouvelle solidarité rurale-urbaine par une taxe pédagogique}

Il faut se donner les moyens d'exercer notre responsabilité et notre solidarité en faveur des milieux ruraux qui rendent d'éminents services (des biens collectifs plus souvent invisibles à toute la société) en leur transférant ainsi des ressources pour qu'ils puissent continuer à le faire pour l'intérêt général; dans nos sociétés, la fiscalité est un outil utilisé pour des telles finalités. Une taxe sur l'alimentation, très basse d'ailleurs, devrait alimenter une caisse qui financerait les actions de développement prévues dans les divers volets de la politique rurale.

Quels sont ces services que rendent gratuitement les ruraux pour le bénéfice de toute la société? Ils sont de plusieurs ordres mais pour parler le langage des spécialistes, on peut dire qu'il s'agit des aménités rurales (un paysage, un environnement sain, un espace de récréation, des produits du terroir, de loisir, etc.) hautement valorisées par les populations urbaines. Or, les conditions économiques sont telles que si on veut encore voir des campagnes vivantes, il faut leur offrir des aides publiques. Car la régulation marchande ne peut pas ou difficilement rétribuer cet immense travail de maintien des paysages ruraux par exemple. Les Québécois reconnaissent que les 1200 villages ruraux qui forment le tissu rural de cette société sont d'une inestimable valeur et font partie de leur patrimoine historique. La situation actuelle est telle qu'il faut des actions publiques pour protéger ce patrimoine, assurer qu'il se maintiendra bien vivant. De là l'idée d'un transfert en retour dans le milieu rural.

Quels sont ces services que rendent gratuitement les ruraux pour le bénéfice de toute la société ? Ils sont de plusieurs ordres mais pour parler le langage des spécialistes, on peut dire qu'il s'agit des aménités rurales (un paysage, un environnement sain, un espace de récréation, des produits du terroir, de loisir, etc.) hautement valorisées par les populations urbaines. Or, les conditions économiques sont telles que si on veut encore voir des campagnes vivantes, il faut leur offrir des aides publiques. Car la régulation marchande ne peut pas ou difficilement rétribuer cet immense travail de maintien des paysages ruraux par exemple.

Mais ce n'est pas la principale raison, car il en est une autre bien plus importante. Les populations urbaines n'ont guère réalisé que les campagnes qui les nourrissent, soumises à la logique marchande, le font avec un retour financier de plus en plus étroit. Sait-on que $90 \%$ du prix d'achat des produits de notre panier d'épicerie se disperse dans la chaîne agroalimentaire et ne remonte pas aux producteurs ruraux et agricoles. Ces derniers ne touchent en moyenne que $10 \%$ du prix payé par le consommateur final. Ce prix, en regard du pouvoir d'achat 
des consommateurs québécois, a tendance à devenir très léger. Au début du siècle, la famille moyenne déboursait plus de 40 à $50 \%$ de son revenu pour l'alimentation (et c'est le cas pour de nombreux pays en développement de nos jours). En moins d'un siècle, cette proportion a chuté à environ $17 \%$. Pourquoi ? Les gains de productivité dans ce secteur, amplifiés avec la mécanisation agricole et les avancées de l'agronomie moderne, ont été considérables et ce sont les consommateurs qui en bénéficient aujourd'hui. Une certaine presse mal informée voudrait nous faire croire que les producteurs agricoles québécois sont des travailleurs inefficaces, gavés de subventions gouvernementales et que cette agriculture nous coûte finalement très cher. Mais en fait, la vérité est que ces subventions - et cela est vrai dans tous les pays dits avancés - qui soutiennent massivement leur agriculture, servent à réduire le coût de l'alimentation, une composante essentielle du coût de la vie selon lequel s'élaborent les demandes salariales des travailleurs urbains.

Les généreuses subventions agricoles ne font que transiter dans le secteur agricole pour aller, en dernière instance, bénéficier aux économies urbaines. Et finalement, ces subventions, présentées aussi comme des mesures de développement rural, consolident davantage le développement urbain et génèrent plutôt un sousdéveloppement rural inacceptable. Sans compter que pour réaliser cet exploit, soit réduire drastiquement le prix du panier d'épicerie auquel on consacre maintenant moins que les loisirs, il a fallu développer un modèle agricole productiviste (qu'on retrouve aussi en forêt) qui pose de sérieuses questions sur sa reproductivité à long terme et sur la qualité des aliments qu'il met dans notre assiette.

Les généreuses subventions agricoles ne font que transiter dans le secteur agricole pour aller, en dernière instance, bénéficier aux économies urbaines. Et finalement, ces subventions, présentées aussi comme des mesures de développement rural, consolident davantage le développement urbain et génèrent plutôt un sous-développement rural inacceptable.

Pour nous, il s'agit d'une taxe symbolique, et même pédagogique, de $1 \%$ sur l'alimentation ${ }^{8}$. Elle serait entièrement dédiée au développement rural. Il faudrait la présenter comme une véritable mesure compensatoire, le marché n'étant pas capable de rémunérer totalement le travail des ruraux qui bénéficie à l'ensemble de la société. Et puisque pour bien des ménages sous le seuil de pauvreté, le coût du panier d'épicerie est un élément budgétaire très important, on pourrait facilement les soustraire de l'application de cette taxe.

Il va sans dire qu'une telle taxe doit être accompagnée d'un effort particulier de sensibilisation de l'opinion publique à la situation rurale. Si ce travail pédagogique est bien fait, au lieu d'être un acte politique suicidaire, il pourrait générer pour le gouvernement un capital de sympathie dans la population; il apparaîtrait alors comme un gouvernement qui ose gouverner, qui assure l'avenir du développement urbain et rural dans le même mouvement, et ce dans la grande tradition de justice redistributive de nos États modernes.

Il va sans dire qu'une telle taxe doit être accompagnée d'un effort particulier de sensibilisation de l'opinion publique à la situation rurale. Si ce travail pédagogique est bien fait, au lieu d'être un acte politique suicidaire, il pourrait générer pour le gouvernement un capital de sympathie dans la population; il apparaîtrait alors comme un gouvernement qui ose gouverner, qui assure l'avenir du développement urbain et rural dans le même mouvement, et ce dans la grande tradition de justice redistributive de nos États modernes.

10. Mettre en ouvre une politique publique appropriée

Après la période fortement interventionniste de l'État providence, nous sommes dans une période qui se caractérise par le retrait de l'État devenu accompagnateur des initiatives locales. L'idée que les communautés rurales puissent se développer seules, sans des aides publiques, est irréaliste et inconséquente. C'est aussi une belle auto-légitimation des pouvoirs publics pour se désengager à un moment où leur engagement est nécessaire. Comme nous l'écrivions dans notre livre Territoire d'avenir, «Si l'État peut se passer du monde rural, le monde rural lui ne peut se passer de l'État. Le politique constitue une instance qui, justement, peut 
jouer un rôle décisif dans la mesure où les ruraux, laissés à eux-mêmes, ne peuvent aménager seuls leur propre avenir». La question du maintien des services publics en milieu rural l'illustre aisément; l'État est directement interpellé dans ses modes de fonctionnement et sa capacité de s'adapter aux réalités rurales.

\section{Quelques objectifs de la politique rurale}

- Soutenir le développement des capacités des collectivités (capacity building)

- Renforcer la gouvernance locale des collectivités locales

- Réduire les disparités (rurales-urbaines) et cultiver les différences rurales

- Maintenir les services de proximité en milieu rural

- Faciliter l'accès aux terres et aux ressources naturelles publiques

- Accélérer la mise en valeur des aménités rurales et préserver l'environnement

- Mettre en œuvre le principe de la solidarité nationale face au rural: passer des programmes d'assistance qui entretiennent la dépendance à une véritable solidarité (développement solidaire).

Dans le tableau ci-haut, je rappelle des propos que j'ai tenus pendant des années sur les orientations d'une politique rurale 9 . Des pas importants ont été franchis dans cette direction et il est faux de prétendre que l'État est insensible à la problématique des milieux ruraux. Le Plan d'action rurale du gouvernement fédéral, suivant la nomination d'un Secrétaire d'État au développement rural et la mise sur pied du Secrétariat rural sont des initiatives importantes. La Politique nationale de la ruralité du Québec 10 introduit aussi une «clause rurale» dans toutes les politiques gouvernementales.

Les gouvernements sont effectivement à la recherche de nouveaux modèles d'intervention pour soutenir ou accompagner les efforts nécessaires afin d'éviter que des pans entiers des économies rurales partent à la dérive. Les pouvoirs publics devront, pour répondre aux attentes des ruraux, agir à deux niveaux assez éloignés. D'un côté, le développement rural requiert que les gouvernements clarifient les grandes politiques macroéconomiques (et sociales) car ce qui inquiète les acteurs économiques, c'est l'absence de règles claires et équita- bles. De l'autre, le développement rural ne sera pas possible sans cette attention de l'État au développement des communautés rurales elles-mêmes par des programmes souples, modulables, décentralisés pour accompagner un effort de développement déjà présent dans les communautés rurales.

\section{Conclusion}

Réussir le développement des communautés rurales suppose de réunir des conditions gagnantes qui ellesmêmes reposent sur un certain nombre de pré-requis et des postulats implicites. Le principal postulat derrière l'énoncé de ces conditions, c'est que la responsabilité du développement appartient d'abord aux ruraux et à leurs communautés, sans nier la responsabilité des pouvoirs publics. Un tel postulat implique un sérieux changement de mentalité dans des territoires où des décennies d'un État providentialiste ont généré une attitude de dépendance et d'attente et où on est déresponsabilisé face à l'aménagement de son propre avenir.

Un des pré-requis, c'est certainement l'accès aux terres et ressources publiques. Souvent, on explique la richesse de certaines zones rurales par des facteurs écologiques (qualité des sols) et économiques (proximité des marchés) en passant sous silence un facteur social majeur: l'accès à la propriété. Les vieux terroirs ruraux de la vallée laurentienne ont été mis en valeur par une agriculture familiale de petite propriété qui s'est transmise au fil des générations. Fortement capitalisée, cette agriculture prospère n'est certes pas étrangère aux vertus de la propriété bien comprise. Il s'en trouve plusieurs aujourd'hui pour dire que nos forêts publiques seraient en un bien meilleur état si ce patrimoine collectif avait été confié à des familles y tenant ces fermes forestières selon le modèle agricole.

La propriété publique est omniprésente en milieu rural et elle y constitue souvent un frein à son développement. Le cas le plus caricatural est celui de la forêt; plusieurs centaines de communautés rurales sont «dépendantes» de la forêt mais cette richesse est appauvrissante pour ces communautés dont les seules retombées sont souvent quelques emplois saisonniers. D'autres territoires ruraux sont le siège des grands barrages hydroélectriques mais les retombées de la mise en valeur de ces ressources rurales leur échappent presque totalement. Ce qui pourrait être considéré comme une expropriation en règle est plutôt perçu, en milieu urbain, 
comme la récolte d'une ressource donnée par la nature, selon une vision providentialiste des ressources naturelles.

\section{La propriété publique est omniprésente} en milieu rural et elle y constitue souvent un frein à son développement. Le cas le plus caricatural est celui de la forêt; plusieurs centaines de communautés rurales sont " dépendantes " de la forêt mais cette richesse est appauvrissante pour ces communautés dont les seules retombées sont souvent quelques emplois saisonniers. D'autres territoires ruraux sont le siège des grands barrages hydroélectriques mais les retombées de la mise en valeur de ces ressources rurales leur échappent presque totalement. Ce qui pourrait être considéré comme une expropriation en règle est plutôt perçu, en milieu urbain, comme la récolte d'une ressource donnée par la nature, selon une vision providentialiste des ressources naturelles.

Finalement, l'exercice auquel nous nous sommes livrés ici repose sur le postulat qu'il n'y a pas de recettes magiques pour assurer le développement des communautés rurales. C'est à chacune de trouver sa recette et une recette valable pour l'une peut ne pas l'être pour une autre. Cependant, ce qui est transmissible, ce sont les processus sociaux qui font qu'on devient capable d'inventer sa recette de développement. Il est aussi implicite que selon la situation locale, certaines conditions évoquées ici sont plus critiques que d'autres. Ainsi, la connaissance de ces conditions n'épargne aucune communauté d'une démarche de diagnostic territorial, qui est la première et nécessaire étape de tout processus de planification du développement.
Notes et références

1 Bruno Jean est titulaire de la Chaire de recherche du Canada en développement rural à l'Université du Québec à Rimouski.

2 Une première esquisse de cette réflexion a été présentée dans le cadre d'une communication à Mentorat 2002 de la Fondation de l'Entrepreneurship, Québec, le 7 février 2002 et lors du Colloque Quel avenir pour les régions au Québec en ce début de millénaire? organisé par l'Université du Québec à Hull le 2 novembre 2001.

3 Voir le site Internet de cette initiative: nre.concordia.ca

4 Ce concept semble, sous le terme de CCB (Community Capacity Building), avoir la faveur de certaines instances publiques canadiennes comme le Secrétariat rural d'Agriculture et Agroalimentaire Canada, Développement des ressources humaines Canada et le Programme de développement des collectivités (Community Futures ou, comme on dit au Québec, les SADC, les Sociétés d'Aide au Développement des Collectivités) qui en font la promotion actuellement.

5 Voir l'article de Mario Carrier et Bruno Jean, «La reconstruction de la légitimité des collectivités rurales: entre gouvernement et gouvernance»dans Mario Carrier et Serge Côté (sous la direction de) (2000). Gouvernance et territoires ruraux. Éléments d'un débat sur la responsabilité du développement. Québec, Presses de l'Université du Québec, Coll. «Sciences régionales ».

6 Solidarité rurale du Québec, Opération des villages prospères (1996). Rapport du groupe de travail, le 9 octobre. Voir: http://www.solidarite-rurale.qc.ca/outils.html

7 Voir: www.crff.ca

8 Les discussions suscitées par la présentation d'une telle idée aux participants du Forum itinérant de l'UQCN sur une agriculture citoyenne de Rimouski le 14 avril 2002 me donnent à penser que la taxe sur l'alimentation pourrait être substituée, comme au Japon, par une taxe sur l'eau consommée dans les villes.

9 Par exemple: Bruno Jean (1994). «Quand l'autre Québec aménage son avenir. Les défis d'une ruralité québécoise moderne » dans l'Action Nationale 94 (9). Montréal, novembre, pp. 1260-1279.

10 Voir à ce sujet: Bruno Jean (2002). «La reconnaissance politique de la ruralité québécoise » dans L'Annuaire du Québec 2003. Montréal, Éditions Fides, pp. 231-241 (Sous la direction de Rock Côté et Michel Venne, en coll. avec Le Devoir). 